\title{
DELETED PRODUCTS OF SPACES WHICH ARE UNIONS OF TWO SIMPLEXES $\left({ }^{1}\right)$
}

\author{
BY \\ W. T. WHITLEY $\left({ }^{2}\right)$
}

\begin{abstract}
If $X$ is a space, the deleted product space, $X^{*}$, is $X \times X-D$, where $D$ is the diagonal. If $Y$ is a space and $f$ is a continuous map from $X$ to $Y$, then $X_{f}^{*}$ is the inverse image of $Y^{*}$ under the map $f \times f$ taking $X \times X$ into $Y \times Y$. In this paper, we investigate the following questions: "What maps $f$ are such that $X_{f}^{*}$ is homotopically equivalent to $X^{*}$ ", and "What maps $f$ are such that $X_{f}^{*}$ is homotopically equivalent to $f(X)^{*}$ ?" If $X$ is the union of two nondisjoint simplexes and $f$ is a simplicial map from $X \times X$ such that $f \mid f(X)$ is one-to-one, we obtain necessary and sufficient conditions for $X_{f}^{*}$ and $f(X)^{*}$ to be homotopically equivalent. If $X$ is the union of nondisjoint simplexes $A$ and $B$ with $\operatorname{dim} B=1+\operatorname{dim}(A \cap B)$, we obtain necessary and sufficient conditions for $X^{*}$ and $X_{f}^{*}$ to be homotopically equivalent if $f$ is in the class of maps mentioned.
\end{abstract}

1. Introduction and notation. If $X$ is a space, the deleted product space, $X^{*}$, of $X$ is $X \times X-D$, where $D$ is the diagonal. If $Y$ is a space and $f$ is a continuous map from $X$ to $Y$, then $X_{f}^{*}$ is the inverse image of $Y^{*}$ under the map $f \times f$ taking $X \times X$ into $Y \times Y$. In [1, p. 236], Brahana asks the question, "What maps $f$ are such that there is a homotopy equivalence between $X_{f}^{*}$ and $X^{*}$ ?"

In this paper, we investigate Brahana's question and the related question, "What maps $f$ are such that there is a homotopy equivalence between $X_{f}^{*}$ and $f(X)^{*}$ ?" If $X$ is a finite polyhedron, let $F(X)$ denote the class of simplicial maps $f$ such that $f(X)$ is a subset of $X$ and such that $f \mid f(X)$ is one-to-one. If $X$ is the union of two nondisjoint simplexes, we are able to obtain complete answers to the second question for all maps $f$ in $F(X)$, and we are able to obtain complete answers to Brahana's question for all maps $f$ in $F(X)$ for a certain subcollection of such spaces.

If $X$ and $Y$ are finite polyhedra and $f$ is a simplicial map from $X$ to $Y$, let $P\left(X^{*}\right)=\bigcup\{r \times s \mid r$ and $s$ are simplexes in $X$ and $r \cap s=\varnothing\}$, and let $P\left(X_{f}^{*}\right)$ $=\bigcup\{r \times s \mid r$ and $s$ are simplexes in $X$ and $f(r) \cap f(s)=\varnothing\}$. In [2, pp. 351-352] Hu has shown that $X^{*}$ and $P\left(X^{*}\right)$ are homotopically equivalent, and in [3, p. 183] Patty has observed that $X_{f}^{*}$ and $P\left(X_{f}^{*}\right)$ are homotopically equivalent.

Presented to the Society, January 23, 1970; received by the editors August 6, 1969 .

AMS 1969 subject classifications. Primary 5525, 5540; Secondary 5530.

Key words and phrases. Deleted products of spaces, unions of two simplexes, homotopy types of deleted products, homology groups of deleted products, simplicial maps on polyhedra, subspace of deleted products determined by simplicial map.

(1) The results of this paper are taken from the author's doctoral dissertation, written under the direction of Dr. C. W. Patty, at Virginia Polytechnic Institute and State University.

$\left.{ }^{2}\right)$ The author's present address is Department of Mathematics, Marshall University, Huntington, West Virginia. 
The symbol $\left\langle v_{0}, \ldots, v_{n}\right\rangle$ will denote the $n$-simplex whose vertices are $v_{0}, \ldots, v_{n}$, and we shall use the circumflex $\hat{v}_{i}$ to indicate that the vertex $v_{i}$ has been deleted. That is, $\left\langle v_{0}, \ldots, \hat{v}_{i}, \ldots, v_{n}\right\rangle$ will denote the $(n-1)$-face of $\left\langle v_{0}, \ldots, v_{n}\right\rangle$ with the vertex $v_{i}$ omitted. The symbol $I$ will denote the closed unit interval $[0,1]$, and $S^{n}=\left\{x \mid x\right.$ is in $E^{n+1}$ with $\left.|x|=1\right\}$. If $f$ is a continuous simplicial map on a finite polyhedron $X$ to a finite polyhedron $Y, \eta_{f}$ will be the map $f \times f$ on $P\left(X^{*}\right)$ (or a specified subspace of $P\left(X^{*}\right)$ ) to $Y \times Y$. If $X$ is a polyhedron, $H_{k}(X), k$ a positive integer, will be the $k$ th homology group of $X$ over the integers $Z$. If $G$ and $H$ are groups, $G+H$ will denote the direct sum of $G$ and $H$. If $X$ and $Y$ are spaces, $X \simeq Y$ will mean that $X$ is homotopically equivalent to $Y$.

The author wishes to express his gratitude to Professor C. W. Patty.

\section{Some preliminary results.}

THEOREM 2.1. Let $X$ be a finite simplicial complex. Let $g: X \rightarrow X$ and $f: g(X) \rightarrow X$ be simplicial maps with the following properties: (1) $f^{2}=f$ and $g^{2}=g$, (2) if $x$ is in $X$, then $x$ and $g(x)$ lie in a common simplex in $X$, and (3) if $x$ is in $g(X)$, then $x$ and $f(x)$ lie in a common simplex in $X$. Then $P\left(X_{f g}^{*}\right)$ is homotopically equivalent to $P\left(f g(X)^{*}\right)$.

Proof. We first show that $(f g)^{2}=f g$. Let $v$ be a vertex of $X$. Since $f g(v)$ is a vertex in $g(X), g f g(v)=f g(v)$ by (1). Then since $f^{2}=f$, it follows that $f g f g(v)=f f g(v)=f g(v)$.

It now follows that $P\left(f g(X)^{*}\right)$ is a subset of $P\left(X^{*}\right)$, and that if

$$
\eta_{f g}=f g \times f g: P\left(X_{f g}^{*}\right) \rightarrow P\left(f g(X)^{*}\right),
$$

then $\eta_{f g} \mid P\left(f g(X)^{*}\right)$ is the identity. Hence, if $\eta_{i}: P\left(f g(X)^{*}\right) \rightarrow P\left(X_{f g}^{*}\right)$ is the injection, $\eta_{f g} \eta_{i}$ is the identity. We shall show that $\eta_{f g}$ and $\eta_{i}$ are homotopy inverses.

Let $(x, y)$ be a point in $P\left(X_{f g}^{*}\right)$. Let $r$ and $s$ be the smallest closed simplexes in $X$ containing $x$ and $y$, respectively. Then $r \times s$ is contained in both $P\left(X_{g}^{*}\right)$ and $P\left(X_{f g}^{*}\right)$. Let $r^{\prime}$ be a simplex in $X$ containing both $x$ and $g(x)$, and let $s^{\prime}$ be a simplex in $X$ containing both $y$ and $g(y)$. Let $r_{1}$ be the face of $r^{\prime}$ consisting of the vertices of $r$ together with the vertices of $g(r) \cap r^{\prime}$. Construct $s_{1}$ in a similar manner from $s, s^{\prime}$, and $g(s)$. Then

$$
\begin{aligned}
f g\left(r_{1}\right) & \subset f g(r) \cup\left[f g(g(r)) \cap f g\left(r^{\prime}\right)\right] \\
& =f g(r) \cup\left[f g(r) \cap f g\left(r^{\prime}\right)\right]=f g(r) .
\end{aligned}
$$

Similarly, $f g\left(s_{1}\right)=f g(s)$, so $r_{1} \times s_{1}$ is a subset of $P\left(X_{f g}^{*}\right)$. Hence, the line joining $(x, y)$ and $(g(x), g(y))$ is a subset of $P\left(X_{f g}^{*}\right)$.

Now since $(g(x), g(y))$ is in $P\left(X_{f g}^{*}\right)$, there exist simplexes $u$ and $w$ in $X$ such that $(g(x), g(y))$ is in $u \times w \subset P\left(X_{f g}^{*}\right)$. We may assume that $u$ and $w$ are the smallest closed simplexes in $X$ containing $g(x)$ and $g(y)$ respectively. Let $u^{\prime}$ be a simplex in $X$ containing both $g(x)$ and $f g(x)$, and let $w^{\prime}$ be a simplex in $X$ containing both $g(y)$ and $f g(y)$. Let $u_{1}$ denote the face of $u^{\prime}$ consisting of the vertices of $u$ together with the vertices of $f g(u) \cap u^{\prime}$. Construct $w_{1}$ in a similar manner from $w, w^{\prime}$, and $f g(w)$. Then as above, $f g\left(u_{1}\right)=f g(u)$ and $f g\left(w_{1}\right)=f g(w)$ since $(f g)^{2}=f g$. Then since 
$u \times w$ is a subset of $P\left(X_{f g}^{*}\right), u_{1} \times w_{1}$ is a subset of $P\left(X_{f g}^{*}\right)$ containing both $(g(x), g(y))$ and $(f g(x), f g(y))$. Hence, the line joining $(g(x), g(y))$ and $(f g(x), f g(y))$ is contained in $P\left(X_{f g}^{*}\right)$.

Define $H: P\left(X_{f g}^{*}\right) \times I \rightarrow P\left(X_{f g}^{*}\right)$ by

$$
H(x, y, t)=(1-2 t)(x, y)+2 t \eta_{g}(x, y) \quad \text { if } 0 \leqq t \leqq \frac{1}{2},
$$

and

$$
H(x, y, t)=(2-2 t) \eta_{g}(x, y)+(2 t-1) \eta_{i} \eta_{f g}(x, y) \quad \text { if } \frac{1}{2} \leqq t \leqq 1 .
$$

From the preceding remarks, it follows that $H$ is a homotopy between $\eta_{i} \eta_{f g}$ and the identity on $P\left(X_{f g}^{*}\right)$. This completes the proof.

Corollary 2.1. Let $X$ be a finite simplicial complex. Let $f: X \rightarrow X$ be a simplicial map such that (1) $f^{2}=f$, and (2) if $x$ is a point of $X$, $x$ and $f(x)$ lie in a common simplex of $X$. Then $P\left(X_{f}^{*}\right)$ is homotopically equivalent to $P\left(f(X)^{*}\right)$.

Proof. Let $f=g$ in Theorem 2.1.

THEOREM 2.2. Let $X$ be a finite simplicial complex, and let $f$ be a map in $F(X)$. Then there is a unique simplicial map $h: X \rightarrow X$ such that (1) $h^{2}=h,(2) h(X)=f(X)$, and (3) $P\left(X_{h}^{*}\right)=P\left(X_{f}^{*}\right)$. Furthermore, $f=f h$.

Proof. Since $f \mid f(X)$ is one-to-one, there is a simplicial map $g: f(X) \rightarrow f(X)$ defined by $g(v)=v^{\prime}$, where $v$ is a vertex in $f(X)$ and $v^{\prime}$ is the unique vertex in $f(X)$ such that $f\left(v^{\prime}\right)=v$. Note that $g=(f \mid f(X))^{-1}$, so $f g$ is the identity on $f(X)$. Let $h=g f$. We shall show that $h$ is the required map.

Since $h^{2}=g f g f=g f=h,(1)$ holds.

Let $v$ be a vertex of $f(X)$. Let $v^{\prime}=f(v)$. Then $v=g\left(v^{\prime}\right)=g f(v)$, so $f(X)$ is a subset of $g f(X)=h(X)$. Clearly $g f(X)$ is a subset of $f(X)$ by definition of $g$, so $h(X)=f(X)$.

Let $r$ and $s$ be simplexes in $X$. Suppose $f(r) \cap f(s)=\varnothing$. If $g f(r) \cap g f(s) \neq \varnothing$, then $f g f(r) \cap f g f(s)=f(r) \cap f(s) \neq \varnothing$, which is a contradiction. Hence, $P\left(X_{f}^{*}\right)$ is contained in $P\left(X_{h}^{*}\right)$. If $g f(r) \cap g f(s)=\varnothing$, then $f g f(r) \cap f g f(s)=f(r) \cap f(s)=\varnothing$ since $f|f(X)=f| h(X)$ is one-to-one. Hence, $P\left(X_{h}^{*}\right)$ is a subset of $P\left(X_{f}^{*}\right)$, so (3) holds.

Since $h=g f$, it follows that $f h=f g f=f$.

To prove uniqueness, suppose $h_{1}$ and $h_{2}$ are two simplicial maps satisfying conditions (1), (2), and (3). Let $v$ be a vertex of $X$. Suppose $h_{1}(v) \neq h_{2}(v)$. Now $h_{1}(v)$ is in $h_{1}(X)=f(X)=h_{2}(X)$. Then since $h_{2}^{2}=h_{2}$, we have $h_{2} h_{1}(v)=h_{1}(v)$. Since $h_{2}(v) \neq h_{1}(v),\left(v, h_{1}(v)\right)$ is in $P\left(X_{h_{2}}^{*}\right)=P\left(X_{h_{1}}^{*}\right)$. However, since $h_{1}^{2}=h_{1}$, we must have $h_{1}(v)=h_{1} h_{1}(v)$, which is a contradiction. Thus, $h_{1}=h_{2}$, so the proof is complete.

Observe that if $X, f$, and $h$ are as in Theorem 2.2, and if $v$ and $v^{\prime}$ are vertices of $X$, then $f(v)=f\left(v^{\prime}\right)$ if and only if $h(v)=h\left(v^{\prime}\right)$.

Patty has shown in [4, Corollary 1] that if $A$ is a $p$-simplex, $p \geqq 1$, then $P\left(A^{*}\right)$ has the homotopy type of $S^{P-1}$. This fact, together with the preceding two theorems, yields the following result. 
Corollary 2.2. Let $X$ be an n-simplex. Let $f$ be a map in $F(X)$. Then $P\left(X^{*}\right)$ is homotopically equivalent to $P\left(X_{f}^{*}\right)$ if and only if $f(X)=X$. In any case, $P\left(X_{f}^{*}\right)$ is homotopically equivalent to $P\left(f(X)^{*}\right)$.

Proof. Let $h$ be the map of Theorem 2.2 associated with $f$. By Theorem 2.1, $P\left(X_{h}^{*}\right)$ is homotopically equivalent to $P\left(h(X)^{*}\right)$. Hence, $P\left(X_{f}^{*}\right)$ is homotopically equivalent to $P\left(f(X)^{*}\right)$. The result then follows from Corollary 1 of [4].

3. The main results. The main results are Theorems 3.1, 3.2, 3.3, and 3.4, the proofs of which require a sequence of lemmas. In this section, we shall let $X=A \cup B$, where $A=\left\langle v_{0}, \ldots, v_{k}, v_{k+1}, \ldots, v_{n}\right\rangle$ and $B=\left\langle v_{0}, \ldots, v_{k}, w_{k+1}, \ldots, w_{m}\right\rangle$ are $n$ - and $m$-simplexes, respectively, such that $A \cap B=\left\langle v_{0}, \ldots, v_{k}\right\rangle, k \geqq 0$, is a proper $k$-face of each.

THEOREM 3.1. Let $f$ be a map in $F(X)$ such that $f(X) \cap(A-B)$ is not empty and $f(X) \cap(B-A)$ is not empty. Then $P\left(X_{f}^{*}\right)$ and $P\left(f(X)^{*}\right)$ have the same homotopy type.

Proof. By Theorem 2.2, we may assume $f^{2}=f$. Let us suppose $w_{m}$ is a vertex in $f(X) \cap(B-A)$ and $v_{n}$ is a vertex in $f(X) \cap(A-B)$. Then since $f^{2}=f$, it follows that $f\left(w_{m}\right)=w_{m}$ and $f\left(v_{n}\right)=v_{n}$. Suppose $f(v)$ is in $A-B$ for some vertex $v$ in $B$. Then $\left\langle v, w_{m}\right\rangle$ is a simplex in $X$, but $\left\langle f(v), f\left(w_{m}\right)\right\rangle=\left\langle f(v), w_{m}\right\rangle$ is not a simplex in $X$. Hence, $f(v)$ is in $B$ if $v$ is in $B$, so $f(B)$ is a subset of $B$. Similarly, $f(A)$ is a subset of $A$. The theorem now follows from Corollary 2.1 .

LEMMA 1. Let $f$ be a map in $F(X)$ such that $f \mid A$ is nonconstant, $f \mid B$ is nonconstant, and $f(X)$ is a subset of $A$. Then $P\left(A_{f}^{*}\right)$ is homotopically equivalent to $P\left(f(A)^{*}\right)$, which has the homotopy type of $S^{P}$; and $P\left(B_{f}^{*}\right)$ is homotopically equivalent to $P\left(f(B)^{*}\right)$, which has the homotopy type of $S^{q}$, where $p+1=\operatorname{dim} f(A)$, and $q+1=\operatorname{dim} f(B)$.

Proof. By Theorem 2.2, we may assume $f^{2}=f$. Then since $f(A)$ is a subset of $A$, $P\left(A_{f}^{*}\right)$ is homotopically equivalent to $P\left(f(A)^{*}\right)$ by Corollary 2.1 .

Since $f(B)$ is a face of $A$, there is a face of $B$, say $B^{\prime}=\left\langle u_{0}, \ldots, u_{q}\right\rangle$ such that $f\left(u_{i}\right) \neq f\left(u_{j}\right), i \neq j$, and such that $f(B)=f\left(B^{\prime}\right)$. Define $h: f(B) \rightarrow B$ to be the simplicial map $h f\left(u_{i}\right)=u_{i}, i=0, \ldots, q$. Then $h: f(B) \rightarrow B^{\prime}$ is a homeomorphism. Then $h f \mid B: B \rightarrow B$ is a continuous simplicial map, and since $h$ is one-to-one, $P\left(B_{f}^{*}\right)$ $=P\left(B_{h f}^{*}\right)$. It is clear that $h f\left|B^{\prime}=h f\right| h f(B)$ is one-to-one. Since $h f(B)$ is contained in $B,(h f \mid B)^{2}=(h f \mid B)$. Hence, $P\left(B_{h f}^{*}\right)$ is homotopically equivalent to $P\left(h f(B)^{*}\right)$ by Corollary 2.1. Then since $h f(B)$ is homeomorphic to $f(B)$, it follows that $P\left(B_{f}^{*}\right)$ is homotopically equivalent to $P\left(f(B)^{*}\right)$.

The remainder of the lemma follows from Corollary 1 of [4] since $f(A)$ and $f(B)$ are simplexes.

LEMMA 2. Let $f: X \rightarrow X$ be a simplicial map such that (1) $f^{2}=f$, (2) $f(X)$ is a subset of $A$, (3) $f\left(v_{n}\right)$ is not in $f(B)$, and (4) $f(B) \neq f(A \cap B)$. Let $D_{1}=\bigcup\{r \times s \mid r$ and 
$s$ are simplexes in $X, f(r) \cap f(s)=\varnothing, r \subset A$, and $s \subset B\}$. Then the sets $D_{1}$ and $D_{1} \cap P\left(A_{f}^{*}\right)$ are contractible.

Proof. We first show that $D_{1}$ is contractible to $\left(v_{n}, v_{0}\right)$.

Let $g: D_{1} \rightarrow D_{1}$ be the linear map defined by $g(v, w)=\left(v_{n}, w\right)$ if $v \in f^{-1} f\left(v_{0}\right)$, and $g(v, w)=(v, w)$ otherwise, where $v$ and $w$ are vertices in $X$. Let $(x, y) \in r \times s \subset D_{1}$. Then $r \subset A$ and $s \subset B$. If $r \cap f^{-1} f\left(v_{0}\right)=\varnothing$, then $g(r \times s)=r \times s \subset D_{1}$. Suppose $r \cap f^{-1} f\left(v_{0}\right) \neq \varnothing$. Since $f\left(v_{n}\right)$ is not in $f(B), f\left(v_{n}\right)$ is not in $f(s)$. Hence, $r \times s \subset r^{\prime} \times s \subset D_{1}$, where $r^{\prime}$ is the face of $A$ consisting of the vertices of $r$ together with $v_{n}$. Then clearly $g(r \times s)=g\left(r^{\prime} \times s\right) \subset r^{\prime} \times s \subset D_{1}$. Hence, in any case, if $(x, y)$ is in $D_{1}$, the line joining $(x, y)$ and $g(x, y)$ is contained in $D_{1}$.

Now we shall show that if $(x, y)$ is in $g\left(D_{1}\right)$, the line joining $(x, y)$ and $\left(v_{n}, v_{0}\right)$ is contained in $D_{1}$. Let $(x, y) \in r \times s \subset g\left(D_{1}\right), r$ and $s$ simplexes in $X$. By definition of $g$, it is clear that $r \cap f^{-1} f\left(v_{0}\right)=\varnothing$. Thus, $r \times s \subset r^{\prime} \times s^{\prime} \subset D_{1}$, where $r^{\prime}$ is the face of $A$ consisting of the vertices of $r$ together with $v_{n}$, and $s^{\prime}$ is the face of $B$ consisting of the vertices of $s$ together with $v_{0}$. Then $\left\{(x, y),\left(v_{n}, v_{0}\right)\right\} \subset r^{\prime} \times s^{\prime} \subset D_{1}$, which proves our assertion.

Let $H: D_{1} \times I \rightarrow D_{1}$ be the map defined by

$$
\begin{aligned}
H(x, y, t) & =(1-2 t)(x, y)+2 \operatorname{tg}(x, y), & & 0 \leqq t \leqq \frac{1}{2}, \\
& =(2-2 t) g(x, y)+(2 t-1)\left(v_{n}, v_{0}\right), & & \frac{1}{2} \leqq t \leqq 1 .
\end{aligned}
$$

Then in view of the preceding remarks, it is clear that $H$ is a homotopy between the constant map $\left(v_{n}, v_{0}\right)$ and the identity on $D_{1}$. Therefore, $D_{1}$ is contractible.

Note that $D_{1} \cap P\left(A_{f}^{*}\right)=\bigcup\{r \times s \mid r$ and $s$ are simplexes in $X, f(r) \cap f(s)=\varnothing$, $r \subset A, s \subset A \cap B\}$. Then by the same arguments as above, $D_{1} \cap P\left(A_{f}^{*}\right)$ is contractible to $\left(v_{n}, v_{0}\right)$. This proves the lemma.

Lemma 3. Let $f$ and $D_{1}$ satisfy the hypotheses of Lemma 2. Let $D_{2}=\bigcup\{s \times r \mid r$ and $s$ are simplexes in $X, f(r) \cap f(s)=\varnothing, r \subset A, s \subset B\}$. Then $P\left(A_{f}^{*}\right)$ is homotopically equivalent to $P\left(A_{f}^{*}\right) \cup D_{1} \cup D_{2}$.

Proof. By arguments similar to those of Lemma 2, we have that $D_{2}$ and $D_{2} \cap P\left(A_{f}^{*}\right)$ are contractible. Since $D_{1}$ and $D_{1} \cap P\left(A_{f}^{*}\right)$ are contractible, $P\left(A_{f}^{*}\right)$ $\simeq P\left(A_{f}^{*}\right) \cup D_{1}$. It is clear that $D_{1} \cap D_{2}=P\left(A_{f}^{*}\right) \cap D_{1} \cap D_{2}=P\left((A \cap B)_{f}^{*}\right)$. Hence, $\left(P\left(A_{f}^{*}\right) \cup D_{1}\right) \cap D_{2}=P\left(A_{f}^{*}\right) \cap D_{2}$, which is contractible. Thus, we have

$$
P\left(A_{f}^{*}\right) \cup D_{1} \cup D_{2} \simeq P\left(A_{f}^{*}\right) \cup D_{1} \simeq P\left(A_{f}^{*}\right) .
$$

LeMmA 4. Let $f: X \rightarrow X$ be a simplicial map such that (1) $f^{2}=f$, (2) $f(X)$ is a subset of $A$, and (3) $f\left(w_{m}\right)$ is not in $f(A \cap B)$, and let $D_{1}$ and $D_{2}$ be as in Lemma 2 and Lemma 3. Then $\left(D_{1} \cup D_{2}\right) \cap P\left(B_{f}^{*}\right)$ is homotopically equivalent to

$$
P\left(\left\langle v_{0}, \ldots, v_{k}, w_{m}\right\rangle_{f}^{*}\right) .
$$

(Note that we are not assuming quite all of the conditions of Lemma 2 on f.) 
Proof. Let $g: X \rightarrow X$ be the simplicial map defined by $g\left(w_{i}\right)=w_{m}, k+1 \leqq i \leqq m$, $g\left(v_{i}\right)=v_{i}, 0 \leqq i \leqq n$. We shall show that $\eta_{g}:\left(D_{1} \cup D_{2}\right) \cap P\left(B^{*}\right) \rightarrow P\left(\left\langle v_{0}, \ldots, v_{k}, w_{m}\right\rangle_{f}^{*}\right)$ is the required homotopy equivalence.

Clearly $P\left(\left\langle v_{0}, \ldots, v_{k}, w_{m}\right\rangle_{f}^{*}\right)$ is a subset of $\left(D_{1} \cup D_{2}\right) \cap P\left(B_{f}^{*}\right)$. It is straightforward to check that $\eta_{g}\left(\left(D_{1} \cup D_{2}\right) \cap P\left(B_{f}^{*}\right)\right)$ is contained in $P\left(\left\langle v_{0}, \ldots, v_{k}, w_{m}\right\rangle_{f}^{*}\right)$ by virtue of condition (3).

Let $\eta_{i}: P\left(\left\langle v_{0}, \ldots, v_{k}, w_{m}\right\rangle_{f}^{*}\right) \rightarrow\left(D_{1} \cup D_{2}\right) \cap P\left(B_{f}^{*}\right)$ be the injection. Then clearly $\eta_{g} \eta_{i}$ is the identity.

Let $(x, y) \in r \times s$, where $r$ and $s$ are simplexes in $X$ such that

$$
r \times s \subset\left(D_{1} \cup D_{2}\right) \cap P\left(B_{f}^{*}\right) .
$$

If both $r$ and $s$ are contained in $A \cap B$, then $\eta_{g}(r \times s)=r \times s$. Suppose $s$ is not contained in $A \cap B$. Then $r$ is in $A \cap B$. Hence, if $s^{\prime}$ is the face of $B$ consisting of the vertices of $s$ together with $w_{m}$, we must have $r \times s \subset r \times s^{\prime} \subset D_{1} \cap P\left(B_{f}^{*}\right)$. Similarly, if $r$ is not contained in $A \cap B$, then $r \times s \subset r^{\prime} \times s \subset D_{2} \cap P\left(B_{f}^{*}\right)$ where $r^{\prime}$ is $r$ with $w_{m}$ adjoined. Clearly $\eta_{g}\left(r \times s^{\prime}\right) \subset r \times s^{\prime}$ (or $\left.\eta_{g}\left(r^{\prime} \times s\right) \subset r^{\prime} \times s\right)$. Hence, in any case, if $(x, y)$ is a point in $\left(D_{1} \cup D_{2}\right) \cap P\left(B_{f}^{*}\right)$, the line joining $(x, y)$ and $\eta_{g}(x, y)$ is contained in $\left(D_{1} \cup D_{2}\right) \cap P\left(B_{f}^{*}\right)$. Thus, the map

$$
H:\left(D_{1} \cup D_{2}\right) \cap P\left(B_{f}^{*}\right) \times I \rightarrow\left(D_{1} \cup D_{2}\right) \cap P\left(B_{f}^{*}\right)
$$

defined by $H(x, y, t)=(1-t)(x, y)+t \eta_{i} \eta_{g}(x, y)$ is a homotopy between $\eta_{i} \eta_{g}$ and the identity. This proves the lemma.

LEMMA 5. Let $f$ be as in Lemma 4. Let $w_{m}$ be a vertex of $B$ such that $f\left(w_{m}\right)$ is not in $f(A \cap B)$. Let $B^{\prime}=\left\langle v_{0}, \ldots, v_{k}, w_{m}\right\rangle$. If $f(B)=f\left(B^{\prime}\right)$, then $P\left(X_{f}^{*}\right)$ is homotopically equivalent to $P\left(f(X)^{*}\right)$.

Proof. Let $X^{\prime}=A \cup B^{\prime}$. Since $f(B)=f\left(B^{\prime}\right)$, it is clear that $f\left(X^{\prime}\right)=f(X)$. We first show that $P\left(X_{f}^{\prime *}\right)$ has the homotopy type of $P\left(f(X)^{*}\right)$.

Let $D_{1}^{\prime}=\bigcup\left\{r \times s \mid r\right.$ is a simplex in $A$, $s$ is a simplex in $B^{\prime}$, and $\left.f(r) \cap f(s)=\varnothing\right\}$. Let $D_{2}^{\prime}=\bigcup\left\{s \times r \mid r\right.$ is a simplex in $A, s$ is a simplex in $B^{\prime}$, and $\left.f(r) \cap f(s)=\varnothing\right\}$. Then since $P\left(B_{f}^{\prime *}\right)$ is a subset of $D_{1}^{\prime} \cup D_{2}^{\prime}$, we have $P\left(X_{f}^{\prime *}\right)=P\left(A_{f}^{*}\right) \cup D_{1}^{\prime} \cup D_{2}^{\prime}$. By Lemma 3, $P\left(A_{f}^{*}\right)$ is homotopically equivalent to $P\left(X_{f}^{\prime *}\right)$. Since $f^{2}=f$ and $f(A)$ is contained in $A$, it follows that $P\left(A_{f}^{*}\right)$ is homotopically equivalent to $P\left(f(A)^{*}\right)$ by Corollary 2.1. However, $f^{2}=f$ and $f(X)$ a subset of $A$ imply that $f(X)=f(A)$. Hence $P\left(X_{f}^{*}\right) \simeq P\left(A_{f}^{*}\right) \simeq P\left(f(X)^{*}\right)$.

To complete the proof, we need to show that $P\left(X_{f}^{*}\right)$ and $P\left(X_{f}^{\prime *}\right)$ have the same homotopy type. Note that $P\left(X_{f}^{\prime *}\right)$ is clearly a subset of $P\left(X_{f}^{*}\right)$. Let $u_{0}, \ldots, u_{q}$ be vertices of $A \cap B$ such that $f\left(u_{i}\right) \neq f\left(u_{1}\right), i \neq j$, and $f\left(\left\langle u_{0}, \ldots, u_{q}\right\rangle\right)=f(A \cap B)$. Then if $v$ is a vertex of $B, v$ is in exactly one of the sets $f^{-1} f\left(u_{0}\right), \ldots, f^{-1} f\left(u_{q}\right), f^{-1} f\left(w_{m}\right)$. Let $g: X \rightarrow X$ be the simplicial map defined by $g(v)=u_{i}, v \in f^{-1} f\left(u_{i}\right) \cap B$, $0 \leqq i \leqq q, g(v)=w_{m}, v \in f^{-1} f\left(w_{m}\right) \cap B$, and $g(v)=v$ otherwise, where $v$ is a vertex in $X$. Note that $g^{2}=g$. 
Suppose $v$ and $v^{\prime}$ are vertices of $X$ with $\left(v, v^{\prime}\right)$ in $P\left(X_{f}^{*}\right)$. Then since $f g(v)=f(v)$ and $f g\left(v^{\prime}\right)=f\left(v^{\prime}\right)$, we see that $\left(g(v), g\left(v^{\prime}\right)\right) \in P\left(X_{f}^{*}\right) \subset P\left(X_{f}^{*}\right)$. Thus, we have $\eta_{g}: P\left(X_{f}^{*}\right) \rightarrow P\left(X_{f}^{* *}\right)$. Note that we also have $\eta_{g}: P\left(X_{f}^{*}\right) \rightarrow P\left(X_{f}^{\prime *}\right) \subset P\left(X_{f}^{*}\right)$ by the above argument. We shall show that $\eta_{g}: P\left(X_{f}^{*}\right) \rightarrow P\left(X_{f}^{\prime *}\right)$ and $\eta_{g}: P\left(X_{f}^{\prime *}\right) \rightarrow P\left(X_{f}^{\prime *}\right)$ are homotopy inverses.

Let $(x, y) \in r \times s \subset P\left(X_{f}^{*}\right), r$ and $s$ simplexes in $X$. If $r$ is in $A$, then $g(r)$ is in $A$; and if $r$ is in $B$, then $g(r)$ is in $B$. Let $r^{\prime}$ be the simplex in $X$ consisting of the vertices of $r$ together with the vertices of $g(r)$, and let $s^{\prime}$ be the simplex in $X$ consisting of the vertices of $s$ together with the vertices of $g(s)$. Then since $f g(r)=f(r)$ and $f g(s)$ $=f(s), r^{\prime} \times s^{\prime} \subset P\left(X_{f}^{*}\right)$ since $r \times s \subset P\left(X_{f}^{*}\right)$. Hence, if $(x, y)$ is in $P\left(X_{f}^{*}\right)$, the line joining $(x, y)$ and $(g(x), g(y))$ is contained in $P\left(X_{f}^{*}\right)$. Therefore, the map $H: P\left(X_{f}^{*}\right) \times I \rightarrow P\left(X_{f}^{*}\right)$, defined by $H(x, y, t)=(1-t)(x, y)+t \eta_{g} \eta_{g}(x, y)$, is a homotopy between $\eta_{g} \eta_{g}$ and the identity on $P\left(X_{f}^{*}\right)$.

To complete the proof, note that if $r$ and $s$ are simplexes in $X$ with $r \times s$ in $P\left(X_{f}^{\prime *}\right)$, then $r$ and $s$ are in $X^{\prime}$, which implies $g(r)$ and $g(s)$ are in $X^{\prime}$. Hence, if $r^{\prime}$ and $s^{\prime}$ are constructed as above, $r^{\prime} \times s^{\prime}$ is contained in $P\left(X_{f}^{\prime *}\right)$. Then the map $H \mid P\left(X_{f}^{\prime *}\right) \times I$ is a homotopy between $\eta_{g} \eta_{g}$ and the identity on $P\left(X_{f}^{\prime *}\right)$. This proves the lemma.

LEMMA 6. Let $f$ be a map in $F(X)$ such that (1) $f\left(v_{n}\right)$ is not in $f(B),(2) f\left(w_{m}\right)$ is not in $f(A \cap B)$, and (3) $f(X)$ is a subset of $A$. Then $P\left(X_{f}^{*}\right)$ and $P\left(f(X)^{*}\right)$ have the same homotopy type if and only if $f(B)=f\left(\left\langle v_{0}, \ldots, v_{k}, w_{m}\right\rangle\right)$.

Proof. By Theorem 2.2, we may assume $f^{2}=f$. Then since $f(A)$ is contained in $A$, it follows that $P\left(A_{f}^{*}\right)$ is homotopically equivalent to $P\left(f(A)^{*}\right)$ by Corollary 2.1 . Since $f^{2}=f$ and $f(X)$ is a subset of $A$, we have $f(X)=f(A)$. Hence, $P\left(f(X)^{*}\right)$ has the homotopy type of $P\left(A_{f}^{*}\right)$. Let $D_{1}$ and $D_{2}$ be as in Lemma 2 and Lemma 3. Then we have $P\left(X_{f}^{*}\right)=P\left(A_{f}^{*}\right) \cup D_{1} \cup D_{2} \cup P\left(B_{f}^{*}\right)$.

If $f(B)=f\left(\left\langle v_{0}, \ldots, v_{k}, w_{m}\right\rangle\right)$, then $P\left(X_{f}^{*}\right)$ is homotopically equivalent to $P\left(f(X)^{*}\right)$ by Lemma 5 .

Suppose $f(B) \neq f\left(\left\langle v_{0}, \ldots, v_{k}, w_{m}\right\rangle\right)$. Then $P\left(B_{f}^{*}\right)$ is not a subset of $D_{1} \cup D_{2}$. By Lemma $1, P\left(A_{f}^{*}\right)$ has the homotopy type of $S^{q}$, and $P\left(B_{f}^{*}\right)$ has the homotopy type of $S^{t}$, where $q+1=\operatorname{dim} f(A)$ and $t+1=\operatorname{dim} f(B)$. By conditions (1) and (3) and the fact that $f^{2}=f$, we must have $q>t$. By Lemma $3, P\left(A_{f}^{*}\right) \simeq P\left(A_{f}^{*}\right) \cup D_{1} \cup D_{2} \simeq S^{q}$. Note that $P\left(A_{f}^{*}\right) \cap P\left(B_{f}^{*}\right)=P\left((A \cap B)_{f}^{*}\right)$, which is a subset of $D_{1} \cap P\left(B_{f}^{*}\right)$. Thus,

$$
\begin{array}{rlrl}
{\left[P\left(A_{f}^{*}\right) \cup D_{1} \cup D_{2}\right] \cap P\left(B_{f}^{*}\right)} & =\left(P\left(A_{f}^{*}\right) \cap P\left(B_{f}^{*}\right)\right) \cup\left[\left(D_{1} \cup D_{2}\right) \cap P\left(B_{f}^{*}\right)\right] \\
& =\left(D_{1} \cup D_{2}\right) \cap P\left(B_{f}^{*}\right) & \\
& \simeq P\left(\left\langle v_{0}, \ldots, v_{k}, w_{m}\right\rangle\right)_{f}^{*}, & & \text { by Lemma 4, } \\
& \simeq S^{r}, & & \text { by Lemma 2, }
\end{array}
$$

where $r+1=\operatorname{dim} f\left(\left\langle v_{0}, \ldots, v_{k}, w_{m}\right\rangle\right)$. Then since $f(B) \neq f\left(\left\langle v_{0}, \ldots, v_{k}, w_{m}\right\rangle\right)$, we must have $r<t$. Hence, $H_{t}\left(P\left(A_{f}^{*}\right) \cup D_{1} \cup D_{2} \cup P\left(B_{f}^{*}\right)\right)=H_{t}\left(P\left(X_{f}^{*}\right)\right)$ is isomorphic 
to $Z$ if $r+1<t$, and is isomorphic to $Z+Z$ if $r+1=t$. However, $H_{t}\left(P\left(f(X)^{*}\right)\right)$ is trivial since $P\left(f(X)^{*}\right)$ has the homotopy type of $S^{q}, q>t$. Hence, $P\left(X_{f}^{*}\right)$ is not homotopically equivalent to $P\left(f(X)^{*}\right)$, so the proof is complete.

Lemma 7. Let $f: X \rightarrow X$ be a simplicial map such that (1) $f^{2}=f$, (2) $f(X)$ is a subset of $A$, (3) $f(X)=f(A)=f(B) \neq f(A \cap B)$, and (4) $f(A \cap B)$ is contained in $A \cap B$. Let $u_{0}, \ldots, u_{p}, \ldots, u_{q}$ be the vertices of $A$ such that $f\left(u_{i}\right) \neq f\left(u_{j}\right), i \neq j$, $0 \leqq i, j \leqq p, u_{i}$ is in $A \cap B$ for $0 \leqq i \leqq p, f(A \cap B)=f\left(\left\langle u_{0}, \ldots, u_{p}\right\rangle\right)$, and $f(A)=f\left(A^{\prime}\right)$ $=A^{\prime}$, where $A^{\prime}=\left\langle u_{0}, \ldots, u_{p}, \ldots, u_{q}\right\rangle$. Let $t_{0}, \ldots, t_{p}, \ldots, t_{q}$ be vertices of $B$ such that $t_{i}=u_{i}$ for $0 \leqq i \leqq p, f\left(t_{i}\right) \neq f\left(t_{j}\right)$ for $i \neq j, 0 \leqq i, j \leqq q$, and $f(B)=f\left(B^{\prime}\right)=f(A)=f\left(A^{\prime}\right)$ $=A^{\prime}$, where $B^{\prime}=\left\langle t_{0}, \ldots, t_{p}, \ldots, t_{q}\right\rangle$. Let $W=A^{\prime} \cup B^{\prime}$. Then $P\left(W_{f}^{*}\right)$ is homotopically equivalent to $P\left(X_{f}^{*}\right)$.

Proof. Note that clearly $P\left(W_{f}^{*}\right)$ is a subset of $P\left(X_{f}^{*}\right)$. Further, if $v$ is a vertex of $A$, there is a unique vertex $u_{i}$ in $A^{\prime}$ such that $f(v)=f\left(u_{i}\right)$, and if $v$ is a vertex in $B$, there is a unique vertex $t_{i}$ in $B^{\prime}$ such that $f(v)=f\left(t_{i}\right)$.

Let $g: X \rightarrow X$ be the simplicial map defined by $g(v)=A^{\prime} \cap f^{-1} f(v)$ if $v$ is a vertex in $A$, and $g(v)=B^{\prime} \cap f^{-1} f(v)$ if $v$ is a vertex in $B$. Then clearly $g^{2}=g, g(A)$ is in $A$, and $g(B)$ is in $B$. Further, since $f g(v)=f(v)$ for each vertex $v$ in $X$, it is easy to see that $\eta_{g}\left(P\left(X_{f}^{*}\right)\right)=P\left(W_{f}^{*}\right)$. We shall show that $\eta_{g}$ is a homotopy equivalence.

Let $\eta_{i}: P\left(W_{f}^{*}\right) \rightarrow P\left(X_{f}^{*}\right)$ be the injection. Since $g \mid W$ is the identity, $\eta_{g} \eta_{i}$ is the identity on $P\left(W_{f}^{*}\right)$.

Let $(x, y)$ be in $P\left(X_{f}^{*}\right)$. Let $r$ and $s$ be simplexes in $X$ such that $(x, y) \in r \times s \subset P\left(X_{f}^{*}\right)$. If $r$ is in $A$, then $g(r)$ is in $A$, and if $r$ is in $B$, then $g(r)$ is in $B$. Let $r^{\prime}$ denote the simplex in $X$ consisting of the vertices of $r$ together with the vertices of $g(r)$, and let $s^{\prime}$ denote the simplex in $X$ consisting of the vertices of $s$ together with the vertices of $g(s)$. Since $f g=f$, it is easy to see that $f(r)=f\left(r^{\prime}\right)$ and $f(s)=f\left(s^{\prime}\right)$. Hence, the line joining $(x, y)$ and $\eta_{g}(x, y)$ is contained in $r^{\prime} \times s^{\prime}$, which, in turn, is contained in $P\left(X_{f}^{*}\right)$. Hence, the map $H: P\left(X_{f}^{*}\right) \times I \rightarrow P\left(X_{f}^{*}\right)$, defined by $H(x, y, t)$ $=(1-t)(x, y)+t \eta_{i} \eta_{g}(x, y)$, is a homotopy between $\eta_{i} \eta_{g}$ and the identity. This completes the proof.

Lemma 8. Suppose $n=m$. Let $f: X \rightarrow X$ be a simplicial map such that (1) $f^{2}=\hat{f}$, and (2) $f(X)=f(A)=f(B)=A$. Let $D_{1}=\bigcup\{r \times s \mid r$ is a simplex in $A, s$ is a simplex in $B$, and $f(r) \cap f(s)=\varnothing\}$. Then $D_{1} \cap P\left(A_{f}^{*}\right)$ is contractible.

Proof. We shall show that $D_{1} \cap P\left(A_{f}^{*}\right)$ contracts to $\left(v_{n}, v_{0}\right)$. Note that under the conditions of the lemma, $f \mid A$ is the identity. Further, it is easy to see that $D_{1} \cap P\left(A_{f}^{*}\right)=\bigcup\{r \times s \mid r$ is a simplex in $A$ and $s$ is a simplex in $A \cap B$ with $r \cap s=\varnothing\}$. Hence, if $r$ and $s$ are simplexes in $X$ with $r \times s$ in $D_{1} \cap P\left(A_{f}^{*}\right)$, then $v_{n}$ is not in $s$, so $r \times s \subset r^{\prime} \times s \subset D_{1} \cap P\left(A_{f}^{*}\right)$, where $r^{\prime}$ is the face of $A$ consisting of the vertices of $r$ together with $v_{n}$. To complete the proof, we now use exactly the same procedure as in the proof of Lemma 2 , contracting $D_{1} \cap P\left(A_{f}^{*}\right)$ to $\left(v_{n}, v_{0}\right)$. 
LemMA 9. Let $f$ be a map in $F(X)$ such that (1) $f(X)$ is contained in $A$, (2) $f(X)$ $=f(A)=f(B)$, and $(3) f(X) \neq f(A \cap B)$. Then $P\left(X_{f}^{*}\right)$ is not homotopically equivalent to $P\left(f(X)^{*}\right)$.

Proof. By Theorem 2.2, we may assume $f^{2}=f$. Then since $f(X)$ is contained in $A$, we have $P\left(A_{f}^{*}\right) \simeq P\left(f(A)^{*}\right)=P\left(f(X)^{*}\right)$ by Corollary 2.1. Clearly

$$
P\left(X_{f}^{*}\right)=P\left(A_{f}^{*}\right) \cup D_{1} \cup D_{2} \cup P\left(B_{f}^{*}\right),
$$

where $D_{1}=\bigcup\{r \times s \mid r$ is a simplex in $A, s$ is a simplex in $B$, and $f(r) \cap f(s)=\varnothing\}$, and $D_{2}=\varnothing\{s \times r \mid r$ is a simplex in $A, s$ is a simplex in $B$, and $f(r) \cap f(s)=\varnothing\}$.

Case I. $f \mid A$ is one-to-one and $f \mid B$ is one-to-one. Note that in this case we must have that $f \mid A$ is the identity and $f(X)=A$ since $f^{2}=f$. We must also assume $n=m$. Then if $n=1$, it is easy to see that $P\left(X_{f}^{*}\right)$ has the homotopy type of four points, while $P\left(f(X)^{*}\right)$ has the homotopy type of two points. Hence, we may assume $n>1$ in what follows.

To complete the proof for this case, we first show that $D_{1}$ and $D_{2}$ are homeomorphic to $P\left(A_{f}^{*}\right)$ by the map $\eta_{f}: D_{1} \rightarrow P\left(A_{f}^{*}\right)$. Since $f^{2}=f$, it is clear that $\eta_{f}\left(D_{1}\right) \subset P\left(f(A)^{*}\right)=P\left(A^{*}\right)=P\left(A_{f}^{*}\right)$. Let $v$ and $v^{\prime}$ be vertices of $A$ with $\left(v, v^{\prime}\right)$ in $P\left(A_{f}^{*}\right)$. There is a unique vertex $w^{\prime}$ in $B$ such that $f\left(w^{\prime}\right)=v^{\prime}$. Hence, $\eta_{f}\left(v, w^{\prime}\right)=\left(v, v^{\prime}\right)$, so $\eta_{f}$ is a surjection. Since $f \mid A$ is the identity and $f \mid B$ is one-to-one, it is easy to see that $\eta_{f} \mid D_{1}$ is an injection, so $\eta_{f}: D_{1} \rightarrow P\left(A_{f}^{*}\right)$ is a homeomorphism. Since $D_{2}$ is homeomorphic to $D_{1}$, we have $D_{1} \simeq D_{2} \simeq P\left(A_{f}^{*}\right)$.

Now by Lemma 1 and the fact that $f(X)=f(A)=f(B)=A$, we have $D_{1} \simeq D_{2}$ $\simeq P\left(A_{f}^{*}\right) \simeq P\left(B_{f}^{*}\right) \simeq S^{n-1}$. By Lemma $8, D_{1} \cap P\left(A_{f}^{*}\right)$ is contractible, and thus $D_{2} \cap P\left(A_{f}^{*}\right)$ is also contractible.

If $m=n=k+1$, then $P\left(B_{f}^{*}\right)$ is contained in $D_{1} \cup D_{2}$, so $P\left(X_{f}^{*}\right)=P\left(A_{f}^{*}\right) \cup D_{1} \cup D_{2}$. In this case, $\operatorname{dim} f(A \cap B)=k=n-1$, so by the proof of Lemma $1, P\left((A \cap B)_{f}^{*}\right)$ has the homotopy type of $S^{n-2}$. Then since

$$
\begin{aligned}
\left(P\left(A_{f}^{*}\right) \cup D_{1}\right) \cap D_{2} & =\left(P\left(A_{f}^{*}\right) \cap D_{2}\right) \cup\left(D_{1} \cap D_{2}\right) \\
& =\left(P\left(A_{f}^{*}\right) \cap D_{2}\right) \cup P\left((A \cap B)_{f}^{*}\right) \\
& =P\left(A_{f}^{*}\right) \cap D_{2},
\end{aligned}
$$

which is contractible, we have $H_{n-1}\left(P\left(X_{f}^{*}\right)\right)=H_{n-1}\left(P\left(A_{f}^{*}\right) \cup D_{1} \cup D_{2}\right)$ isomorphic to $Z+Z+Z$.

Suppose $n>k+1$. Then

$$
\begin{aligned}
\left(P\left(A_{f}^{*}\right) \cup D_{1} \cup D_{2}\right) \cap P\left(B_{f}^{*}\right) & =\left(P\left(A_{f}^{*}\right) \cap P\left(B_{f}^{*}\right)\right) \cup\left(\left(D_{1} \cup D_{2}\right) \cap P\left(B_{f}^{*}\right)\right) \\
& =P\left((A \cap B)_{f}^{*}\right) \cup\left(\left(D_{1} \cup D_{2}\right) \cap P\left(B_{f}^{*}\right)\right) \\
& =\left(D_{1} \cup D_{2}\right) \cap P\left(B_{f}^{*}\right) \\
& \simeq P\left(\left\langle v_{0}, \ldots, v_{k}, w_{n}\right\rangle_{f}^{*}\right) \simeq S^{k}
\end{aligned}
$$

by Lemma 4 and Lemma 1 . Then since $k<n-1$, we still have $H_{n-1}\left(P\left(X_{f}^{*}\right)\right)$ $=H_{n-1}\left(P\left(A^{*}\right) \cup D_{1} \cup D_{2} \cup P\left(B_{f}^{*}\right)\right)$ isomorphic to a direct summand of $Z+Z+Z$. 
However, since $H_{n-1}\left(P\left(f(X)_{f}^{*}\right)\right)=H_{n-1}\left(P\left(A_{f}^{*}\right)\right)$ is isomorphic to $Z$, we conclude that $P\left(X_{f}^{*}\right)$ is not homotopically equivalent to $P\left(f(X)_{f}^{*}\right)$.

Case II. We now drop the assumption that $f \mid A$ is one-to-one and $f \mid B$ is oneto-one.

Case II(i). $f(A \cap B)$ is a subset of $A \cap B$. Let $u_{0}, \ldots, u_{p}, \ldots, u_{q}$ be the vertices of $A$ such that $f\left(u_{i}\right) \neq f\left(u_{j}\right)$ for $i \neq j, 0 \leqq i, j \leqq q, u_{i}$ is a vertex of $A \cap B$ for $0 \leqq i \leqq p$, $f\left(\left\langle u_{0}, \ldots, u_{p}\right\rangle\right)=f(A \cap B)$, and $f(A)=f\left(A^{\prime}\right)=A^{\prime}$, where $A^{\prime}=\left\langle u_{0}, \ldots, u_{p}, \ldots, u_{q}\right\rangle$. Note that by conditions (2) and (3) of the lemma, $q\rangle p$. Let $B^{\prime}=\left\langle t_{0}, \ldots, t_{p}, \ldots, t_{q}\right\rangle$, where $t_{i}$ is a vertex of $B$ for $0 \leqq i \leqq q, t_{i}=u_{i}$ for $0 \leqq i \leqq p$, and $f\left(t_{i}\right)=u_{i}$ for $0 \leqq i \leqq q$. Let $W=A^{\prime} \cup B^{\prime}$. Then clearly $f \mid A^{\prime}$ is one-to-one, $f \mid B^{\prime}$ is one-to-one, and $f(W)=A^{\prime}$. Then by Case I, $P\left(W_{f}^{*}\right)$ is not homotopically equivalent to $P\left(f(W)^{*}\right)$. Since $f(X)=f(A)=A^{\prime}$, we have $f(W)=f(X)$. By Lemma $7, P\left(X_{f}^{*}\right)$ is homotopically equivalent to $P\left(W_{f}^{*}\right)$, so in this case, $P\left(X_{f}^{*}\right)$ does not have the homotopy type of $P\left(f(X)^{*}\right)$.

Case II(ii). $f(A \cap B)$ is not a subset of $A \cap B$. Let $u_{0}, \ldots, u_{p}$ be vertices of. $A \cap B$ such that $f\left(u_{i}\right) \neq f\left(u_{j}\right)$ for $i \neq j, 0 \leqq i, j \leqq p$, and $f(A \cap B)=f\left(\left\langle u_{0}, \ldots, u_{p}\right\rangle\right)$. Consider the simplicial map $g: X \rightarrow X$, defined by $g(v)=f(v)$ if $v$ is not in $f^{-1} f(A \cap B)$, and $g(v)=u_{i}$ if $v$ is in $f^{-1} f\left(u_{i}\right), v$ a vertex of $X$. Clearly $g^{2}=g, P\left(X_{f}^{*}\right)$ $=P\left(X_{g}^{*}\right)$, and $f(X)$ is homeomorphic to $g(X)$. By Case II $(\mathrm{i}), P\left(X_{g}^{*}\right)$ is not homotopically equivalent to $P\left(g(X)^{*}\right)$, so the lemma follows.

Observe that we have shown in the preceding lemma that if $\operatorname{dim} f(X)=1, P\left(X_{f}^{*}\right)$ has the homotopy type of four points, while $P\left(f(X)^{*}\right)$ has the homotopy type of two points. If $\operatorname{dim} f(X)=q>1$, we have shown that $H_{q-1}\left(P\left(X_{f}^{*}\right)\right)$ is a direct summand of $Z+Z+Z$.

LemMA 10. Let $f: X \rightarrow X$ be a simplicial map such that $f^{2}=f, f(B)=f(A \cap B)$, and $f(X)$ is contained in $A$. Then $P\left(X_{f}^{*}\right)$ is homotopically equivalent to $P\left(f(X)^{*}\right)$.

Proof. Let $u_{0}, \ldots, u_{p}$ be vertices of $A \cap B$ such that $f\left(u_{i}\right) \neq f\left(u_{j}\right)$ for $i \neq j$, $0 \leqq i, j \leqq p, f\left(\left\langle u_{0}, \ldots, u_{p}\right\rangle\right)=f(B)$, and $A \cap B \cap f(X)=\left\langle u_{0}, \ldots, u_{p}\right\rangle \cap f(X)$. Then if $v$ is a vertex of $B$, it follows that $v$ is in exactly one of the sets $f^{-1} f\left(u_{i}\right), 0 \leqq i \leqq p$.

Let $g: X \rightarrow X$ be the simplicial map defined by $g(v)=u_{i}$ if $v$ is a vertex in $f^{-1} f\left(u_{i}\right) \cap B$, and $g(v)=v$ if $v$ is a vertex in $A-B$. Then clearly $g^{2}=g, g(B)$ is a subset of $A \cap B$, and $g(A)$ is a subset of $A$.

Let $h: g(X) \rightarrow X$ be the simplicial map defined by $h(v)=f(v), v$ a vertex of $g(X)$. Then since $f^{2}=f$, we must have $h^{2}=h$. Since $g(X)$ contains $f(X)$, every point $x$ of $g(X)$ is in some simplex in $X$ containing $h(x)$.

Thus, $g$ and $h$ satisfy Theorem 2.1 , so $P\left(X_{h g}^{*}\right)$ is homotopically equivalent to $P\left(h g(X)^{*}\right)$. Since $h g=f$, the lemma follows.

THEOREM 3.2. Let $f$ be a map in $F(X)$ such that $f(X)$ is contained in $A$. Then $P\left(X_{f}^{*}\right)$ is homotopically equivalent to $P\left(f(X)^{*}\right)$ if and only if $f$ satisfies at least one of the following conditions: (1) $f(B)=f(A \cap B)$, or (2) $f(B)=f\left(\left\langle v_{0}, \ldots, v_{k}, w_{i}\right\rangle\right)$ for some $i, k+1 \leqq i \leqq m$ and $f(B) \neq f(A)$. 
Proof. By Theorem 2.2, we may assume $f^{2}=f$. If (1) holds, $P\left(X_{f}^{*}\right)$ is homotopically equivalent to $P\left(f(X)^{*}\right)$ by Lemma 10 .

Suppose (1) fails. Since $f^{2}=f$ and $f(X)$ is contained in $A, f(B)$ is contained in $f(A)$. If, in addition, $f(B) \neq f(A)$, there is a vertex $v_{j}$ in $A$ such that $f\left(v_{j}\right)$ is not in $f(B)$. Necessarily $k+1 \leqq j \leqq n$.

Now suppose (1) fails but (2) holds. Then $P\left(X_{f}^{*}\right)$ is homotopically equivalent to $P\left(f(X)^{*}\right)$ by Lemma 6.

Finally, suppose both (1) and (2) fail. If $f(B) \neq f(A)$ and $f(B) \neq f\left(\left\langle v_{0}, \ldots, v_{k}, w_{i}\right\rangle\right)$ for every $i, k+1 \leqq i \leqq m$, then since $f(B)$ is a subset of $f(A)$ with $f(B) \neq f(A)$, and $f(B) \neq f(A \cap B)$ by the failure of (1), $P\left(X_{f}^{*}\right)$ is not homotopically equivalent to $P\left(f(X)^{*}\right)$ by Lemma 6 . If $f(B)=f(A)$, then $P\left(X_{f}^{*}\right)$ is not homotopically equivalent to $P\left(f(X)^{*}\right)$ by Lemma 9 . This completes the proof.

Obviously dual conditions hold in Theorem 3.2 in case $f(X)$ is contained in $B$. Hence Theorems 3.1 and 3.2 give us all maps $f$ in $F(X)$ such that $P\left(X_{f}^{*}\right)$ and $P\left(f(X)^{*}\right)$ have the same homotopy type. Also, in the cases where $P\left(X_{f}^{*}\right)$ and $P\left(f(X)^{*}\right)$ are not homotopically equivalent, the proofs of Lemmas 6 and 9 give us information about the homology groups of $P\left(X_{f}^{*}\right)$.

We are able to use Theorems 3.1 and 3.2 to answer Brahana's question for the maps $f$ in $F(X)$ on a subcollection of the spaces which are unions of two simplexes.

The following lemma is a special case of Theorem 5 of [5].

LEMMA 11. If $X=A \cup B$, where $A$ is an n-simplex and $B$ is an m-simplex with $A \cap B$ an $(m-1)$-face of each, $m \geqq 1$, then $X^{*}$ is homotopically equivalent to $A^{*}$.

Theorem 3.3. Let $X=A \cup B$, where $A=\left\langle v_{0}, \ldots, v_{m-1}, v_{m}, \ldots, v_{n}\right\rangle$ is an $n$ simplex and $B=\left\langle v_{0}, \ldots, v_{m-1}, w_{m}\right\rangle$ is an m-simplex such that $A \cap B=\left\langle v_{0}, \ldots, v_{m-1}\right\rangle$ is an $(m-1)$-face of each, with $n>m$. Let $f$ be a map in $F(X)$. Then $P\left(X^{*}\right)$ is homotopically equivalent to $P\left(X_{f}^{*}\right)$ if and only if either (1) $f(X)=X$, or (2) $f(X)=A$.

Proof. If (1) holds, the result is trivial. If (2) holds, we can infer from Theorem 3.2 that $P\left(X_{f}^{*}\right)$ is homotopically equivalent to $P\left(f(X)^{*}\right)=P\left(A^{*}\right)$. By Lemma 11 , we have $P\left(A^{*}\right)$ homotopically equivalent to $P\left(X^{*}\right)$, so that the result follows.

Suppose neither condition holds. First suppose $f(X) \cap(A-B) \neq \varnothing$ and $f(X) \cap(B-A) \neq \varnothing$. Then by Theorem 3.1, it follows that $P\left(X_{f}^{*}\right)$ is homotopically equivalent to $P\left(f(X)^{*}\right)$. But $P\left(f(X)^{*}\right) \simeq P\left((A \cap f(X))^{*}\right)$ by Lemma 11. Since $f(X) \neq X$ but $w_{m}$ is in $f(X)$, it follows that $A \cap f(X)$ is a proper face of $A$. Then $P\left((A \cap f(X))^{*}\right) \nsucceq P\left(A^{*}\right) \simeq P\left(X^{*}\right)$ since $A$ and $A \cap f(X)$ are simplexes. Thus, $P\left(X_{f}^{*}\right)$ is not homotopically equivalent to $P\left(X^{*}\right)$ in this case.

Next, suppose $f(X)$ is a subset of $A$. Then $f(X)$ is a proper face of $A$ since $f(X) \neq A$. If either of the conditions of Theorem 3.2 holds, $P\left(X_{f}^{*}\right)$ is homotopically equivalent to $P\left(f(X)^{*}\right)$. However, $P\left(f(X)^{*}\right) \not \Perp P\left(A^{*}\right) \simeq P\left(X^{*}\right)$ by Corollary 1 of [4]. Suppose both conditions of Theorem 3.2 fail. Note that we must then have $f(A)$ $=f(B)$ in this case. Then by the proof of Lemma 9, either $P\left(X_{f}^{*}\right)$ has the homotopy type of four points, or $H_{q}\left(P\left(X_{f}^{*}\right)\right)$ is isomorphic to a direct summand of $Z+Z+Z$ 
for some $q \geqq 2$. However, since $P\left(A^{*}\right)$ has the homotopy type of $S^{m-1}$ by Corollary 1 of [4], $H_{q}\left(P\left(A^{*}\right)\right)$ is either trivial or isomorphic to $Z$ for each integer $q \geqq 1$, and $P\left(A^{*}\right)$ does not have the homotopy type of four points. Hence, $P\left(X_{f}^{*}\right) \not P P\left(A^{*}\right)$ $\simeq P\left(X^{*}\right)$.

Finally, suppose $f(X)$ is a subset of $B$. The dual conditions of Theorem 3.2 applied to this situation state that $\left(1^{\prime}\right) f(A)=f(A \cap B)$, or $\left(2^{\prime}\right) f(A)=$ $f\left(\left\langle v_{0}, \ldots, v_{m-1}, v_{j}\right\rangle\right)$ for some $j$ such that $m \leqq j \leqq n$, and $f(B) \neq f(A)$. If either $\left(1^{\prime}\right)$ or $\left(2^{\prime}\right)$ holds, $P\left(X_{f}^{*}\right)$ is homotopically equivalent to $P\left(f(X)^{*}\right)$. Since $f(X)$ is a face of $B$ and $\operatorname{dim} B<\operatorname{dim} A$, it follows from Corollary 1 of [4] that $P\left(f(X)^{*}\right)$ is not homotopically equivalent to $P\left(A^{*}\right)$. Since $P\left(A^{*}\right)$ is homotopically equivalent to $P\left(X^{*}\right)$, we conclude that $P\left(X_{f}^{*}\right)$ is not homotopically equivalent to $P\left(X^{*}\right)$. If both $\left(1^{\prime}\right)$ and $\left(2^{\prime}\right)$ fail, it is straightforward to show that we must have $f(A)=f(B)$ since $f(X)$ is contained in $B$. In this case, the proof that $P\left(X_{f}^{*}\right)$ is not homotopically equivalent to $P\left(X^{*}\right)$ is analogous to the case when $f(X)$ is contained in $A$.

The final theorem treats the case when $n=m$, which was omitted in Theorem 3.3.

THEOREM 3.4. Let $X=A \cup B$, where

$$
A=\left\langle v_{0}, \ldots, v_{n-1}, v_{n}\right\rangle \text { and } B=\left\langle v_{0}, \ldots, v_{n-1}, w_{n}\right\rangle
$$

are two $n$-simplexes such that $A \cap B=\left\langle v_{0}, \ldots, v_{n-1}\right\rangle$ is an $(n-1)$-face of each. Let $f$ be a nonconstant map in $F(X)$. Then $P\left(X_{f}^{*}\right)$ is homotopically equivalent to $P\left(X^{*}\right)$ if and only if $f$ satisfies one of the following conditions: (1) $f(X)=X,(2) f(X)=A$ and $f\left(w_{n}\right)$ is in $f(A \cap B)$, or (3) $f(X)=B$ and $f\left(v_{n}\right)$ is in $f(A \cap B)$.

Proof. By Theorem 2.2, we may assume $f^{2}=f$. If (1) holds, the result is trivial. If either (2) or (3) holds, $P\left(X_{f}^{*}\right)$ is homotopically equivalent to $P\left(f(X)^{*}\right)$ by condition (1) of Theorem 3.2. Since either $f(X)=A$ or $f(X)=B$, we can conclude that $P\left(X_{f}^{*}\right) \simeq P\left(A^{*}\right) \simeq P\left(B^{*}\right) \simeq P\left(X^{*}\right)$.

Suppose all three conditions of the corollary fail. First, suppose $f(X) \cap(A-B) \neq \varnothing$ and $f(X) \cap(B-A) \neq \varnothing$. Then by Theorem 3.1,P(X $\left.X_{f}^{*}\right)$ has the homotopy type of $P\left(f(X)^{*}\right)$. Also, $f(X)$ is the union of two $k$-simplexes with a common $(k-1)$-face for some $k \geqq 1$. Since $f(X) \neq X$, we must have $P\left(f(X)^{*}\right) \simeq S^{k-1} \not S^{n-1} \simeq P\left(X^{*}\right)$ by Lemma 11 and Corollary 1 of [4], so $P\left(X_{f}^{*}\right)$ is not homotopically equivalent to $P\left(X^{*}\right)$ in this case.

Next, suppose $f(X)$ is contained in $A$. If $f\left(w_{n}\right)$ is in $f(A \cap B)$, then we have $f(B)=f(A \cap B)$; so by condition (1) of Theorem 3.2, it follows that $P\left(X_{f}^{*}\right)$ is homotopically equivalent to $P\left(f(X)^{*}\right)$. Since $f(X) \neq A, f(X)$ is a proper face of $A$, so $P\left(f(X)^{*}\right)$ is not homotopically equivalent to $P\left(A^{*}\right)$ by Corollary 1 of [4]. Hence, we have $P\left(X_{f}^{*}\right) \simeq P\left(f(X)^{*}\right) \not P\left(A^{*}\right) \simeq P\left(X^{*}\right)$.

Suppose $f(X)$ is contained in $A$ and $f\left(w_{n}\right)$ is not in $f(A \cap B)$. Since $f^{2}=f$, we must have $f(A)=f(B)$. Then by the proof of Lemma 9, since $f(X)=f(A)=f(B)$ $\neq f(A \cap B)$, either $P\left(X_{f}^{*}\right)$ has the homotopy type of four points, or $H_{q}\left(P\left(X_{f}^{*}\right)\right)$ is isomorphic to a direct summand of $Z+Z+Z$ for some integer $q \geqq 1$. However, since 
$P\left(X^{*}\right) \simeq P\left(A^{*}\right) \simeq S^{n-1}$, it is clear that $P\left(X_{f}^{*}\right)$ is not homotopically equivalent to $P\left(X^{*}\right)$.

If $f(X)$ is contained in $B$, the proof is analogous to the case when $f(X)$ is contained in $A$, so the proof is complete.

\section{BIBLIOGRAPHY}

1. T. R. Brahana, On a class of isotopy invariants, Topology of 3-Manifolds and Related Topics (Proc. Univ. of Georgia Inst., 1961), Prentice-Hall, Englewood Cliffs, N. J., 1962, pp. 235-237. MR 25 \#5514.

2. S. T. Hu, Isotopy invariants of topological spaces, Proc. Roy. Soc. London Ser. A 255 (1960), 331-366. MR 22 \#4064.

3. C. W. Patty, Isotopy invariants of trees, Duke Math. J. 31 (1964), 183-197. MR 28 \#2548.

4. - Polyhedra whose deleted products have the homotopy type of the n-sphere, Duke Math. J. 36 (1969), 233-236. MR 40 \#2076.

5. - Deleted products with homotopy types of spheres, Trans. Amer. Math. Soc. 147 (1970), 223-240.

Virginia Polytechnic Institute and State University,

BLACKSBURG, Virginia 24061 